\title{
A fluorinated 2D magnetic coordination polymer
}

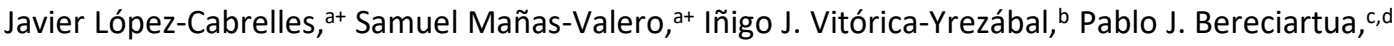

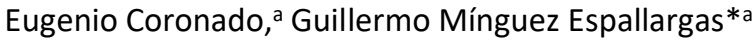

\begin{abstract}
Herein we show the versatility of coordination chemistry to design and expand a family of 2D materials by incorporating $\mathrm{F}$ groups at the surface of the layers. Through of use of a prefuntionalized organic linker with $F$ groups, it is possible to achieve a layered magnetic material based on $\mathrm{Fe}(\mathrm{II})$ centers that are chemically stable in open air, contrary to the known $2 \mathrm{D}$ inorganic magnetic materials. The high quality of the single crystals and their robustness allow to fabricate $2 \mathrm{D}$ molecular materials by micromechanical exfoliation, preserving the crystalline nature of these layers together with the desired functionalization.
\end{abstract}

+ equally contributed

\section{Introduction}

Functionalization of two-dimensional (2D) materials, such as graphene or transition metal dichalcogenides, is an important feature to tune the physical properties of 2D materials, from stability to processability, with the aim of improving their properties. The most common way to successfully functionalize a 2D material is arguably through reactions occurring in solution. ${ }^{1,2}$ However, as typical 2D materials are very inert compounds from a chemical point of view, the covalent functionalization process requires a lot of energy. Therefore, it is normally an uncontrolled step, thus causing defects, low degree of functionalization and attachment of molecules in random positions. Non-covalent functionalization is also an alternative approach, but it typically lacks long-range order, thus resulting in a material more similar to a composite. Examples of high degree of covalent functionalization in graphene ${ }^{3,4}$ and other 2D materials ${ }^{5-7}$ have been reported in the recent years, revealing the need of very particular conditions for a successful incorporation of different molecular moieties.

To overcome this problem, the use of functionalized precursors can be an appropriate solution. ${ }^{8}$ For this reason, fluorographene (FG), a perfluorinated hydrocarbon, took interest in the community due to its reactivity and the possibility to form several graphene derivatives through nucleophilic substitution with high degree of conversion. ${ }^{9}$

\footnotetext{
a. Instituto de Ciencia Molecular (ICMol), Universidad de Valencia, c/Catedrático José Beltrán, 2, 46980 Paterna, Spain.

b. School of Chemistry, University of Manchester, Oxford Road, Manchester M13 GPL, UK.

c. Instituto de Tecnología Química (UPV-CSIC), Universitat Politècnica de València-Consejo Superior de Investigaciones Cientificas, Av. de los Naranjos, $s / n, 46022$ Valencia, Spain.

d. Present address: Deutsches Elektronen-Synchrotron (DESY), Notkestraße 85, 22607 Hamburg, Germany.
}

In this context, coordination chemistry, and reticular design in particular, has given rise to a new route for the functionalization of 2D materials thanks to its greater chemical versatility and tunability, yielding to higher degrees of functionalization, if compared with those achieved in purely inorganic materials. ${ }^{10}$ Thus, the interest in finding $2 \mathrm{D}$ molecular analogues has increased, as they can be chemically tuned by the proper chemical design. ${ }^{11}$ The molecular nature of layered coordination polymers (LCPS) permits, a priori, the incorporation of different functionalization onto the 2D molecular material. This is a common approximation in the field of coordination polymers (CPs), including Metal-Organic Frameworks (MOFs). These are crystalline materials that can be functionalized in a post-synthetic manner in solution. ${ }^{12-14}$ However, the hybrid composition of these coordination compounds allows also to modify the organic linker before the synthesis of the material in order to incorporate a desired functional group, a feature that is impossible, or extremely difficult, to achieve in inorganic materials. In particular, the first example was developed by Yaghi and co-workers using the synthesis of several isoreticular compounds with different functional groups based on the well-known MOF-5. ${ }^{15}$ That can be extended for more systems, as is the case of MOF-74, which can be prepared with different functional groups such as $\mathrm{CH}_{3}$ or $\mathrm{NH}_{2}$ by just using a pre-functionalized organic linker. ${ }^{16}$ This strategy preserves the crystal structure but modifies the physical properties, as for example the $\mathrm{CO}_{2}$ affinity of the pores.

Thus, this approach commonly used in MOFs can serve to circumvent the problems of functionalization of 2D materials. In addition, the possibility of avoiding solvents to obtain the exfoliated material, i.e., combining the synthesis of a prefunctionalized bulk layered material followed by its dry micromechanical exfoliation, would allow to obtain 2D materials with long-range functionalization order.

We have previously used this approach for designing robust 2D molecular materials, presenting an unprecedented 
chemical/physical tunability ${ }^{17}$ and suitable to be integrated into mechanical resonators. ${ }^{8}$ This approach is based on the easy synthesis of pre-functionalized bulk layered materials that are micromechanically exfoliated. Thus, one can obtain an isoreticular family of 2D molecular materials, presenting different magnetic properties or wettability. Herein, we describe the synthesis and characterization of the fluorinated isoreticular analogue of this family, MUV-1-F, a 2D coordination polymer analogous to fluorographene, with long-range magnetic order and well-defined layered crystals, appropriate to prepare 2D magnetic molecular materials.

\section{Results and discussion}

The solvent-free reaction of 5-fluorobenzimidazole (FbimH) and ferrocene, adapting a previously described method for the preparation of iron azolates, ${ }^{8,18}$ yields colorless crystals of around $400 \mu \mathrm{m}$ in size (Fig. S1). Structure determination through single crystal $X$-ray diffraction reveals a laminar material of formula [Fe(Fbim) ${ }_{2}$, denoted as MUV-1-F (MUV = Material of the University of Valencia), consisting in distorted tetrahedral $\mathrm{Fe}(\mathrm{II})$ centers connected by $\mathrm{Fbim}^{-}$bridges, thus forming a neutral layered coordination polymer extended in the $a b$ plane (Fig. 1a), as also found in the isostructural systems MUV-1-X. ${ }^{8}$ The inner part of the layers, i.e. the core, is composed by $\mathrm{Fe}$ (II) centres which are "sandwiched" by benzimidazoles, which serve as an organic protection, resulting in a stable and robust material. In each layer the Fe(II) centers form a square lattice with a metal-metal distance of $5.9 \AA$ (Fig. S2a). The metal centers are connected via benzimidazolate, which coordinate with the two nitrogen heteroatoms, each to a different $\mathrm{Fe}(\mathrm{II})$, with a Fe-N bond distance of 2.036(1) A. These bonds define the short pathway Fe-N-C-N-Fe. The ligands which connect these metallic nodes shape an angle of $47.7^{\circ}$ between the $a b$ plane formed by the square lattice and the tilt of the ligand (Fig. S2b). The position of the fluorine atom in the ligand is indistinguishable between the 5 th and 6 th position due to the deprotonation of the benzimidazole. These two positions provoke a disorder in the crystal structure, originating an infinite number of possibilities for the stacking of the layers.

The neutral layers weakly interact with each other through van der Waals interactions ( $F \cdots F$ interactions of $2.60 \AA$ ). MUV1-F presents a layered morphology, as also found in the isoreticular compounds MUV-1-X,8 regardless of the different functional group, which do not influence the layered crystal growth. This is clearly observed in the SEM images (Fig. 2c).

MUV-1-F shows a high thermal stability until decomposition at around $350{ }^{\circ} \mathrm{C}$ (Fig. 2b). Importantly, the functional group of this material forms part of the organic ligand and, as thermogravimetric analysis measurement shows, there is no mass loss due to the detachment of the functional group, as observed in other 2D materials, where a mass loss corresponding to the extrusion of the functional groups is observed.5,19 The functional groups are located at the surface, thus playing an active role in the chemical behavior of the molecular interface. In this sense, the use of halogen groups enhances the chemical stability of the materials due to their high hydrophobicity, ${ }^{20}$ preventing hydrolysis of sensible materials, typical of $\mathrm{Fe}(\mathrm{II})$ compounds. This behaviour was measured by continuous contact angle (Fig. S3), presenting values of contact angles of $135^{\circ}$ (hydrophobic behaviour).

Fig. 1. a) The layered structure composed by neutral layers.

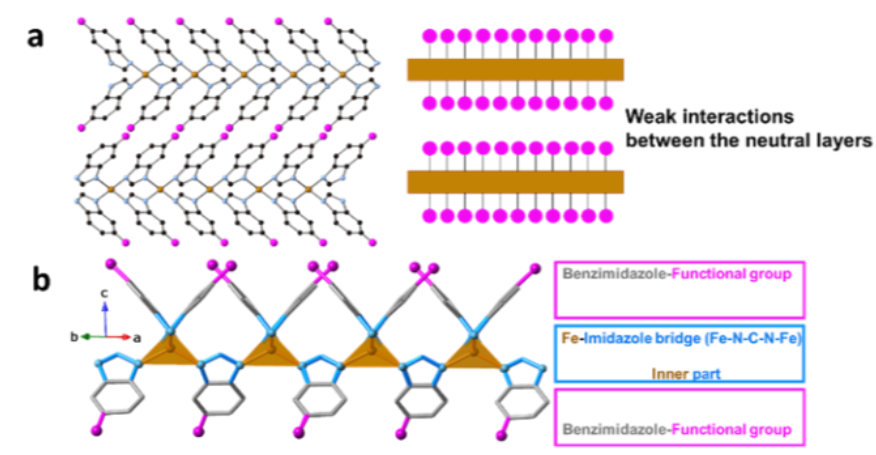

b) The "sandwich" structure for the MUV-1-F layers, formed by a core composed by the Fe(II) centers and imidazole bridges, locating the functionalities in the surface.

The magnetic properties for the MUV-1-F were investigated in order to examine the influence of the different functional groups in the magnetic ordering. As the functional groups are relatively far from the metal centers and the metal-ligand bridge, the electronic properties are not expected to be affected by the different functional groups compared to the reported MUV-1-X family (Fig. 1b). Fig. 3 shows the thermal dependence of the magnetic susceptibility, exhibiting antiferromagnetic interactions, confirmed by a negative CurieWeiss temperature $(\theta=-114 \pm 3 \mathrm{~K})$, and a sharp peak in $\chi$ with a maximum at ca. $19 \mathrm{~K}$, indicating the phase transition to a canted antiferromagnetic ordering. This magnetic scenario is confirmed by the temperature dependence of the ac magnetic susceptibility (Fig. 3b), where an out-of-plane ac signal is observed below $20 \mathrm{~K}$. The dc data is fitted to the Lines model for a quadratic-layer antiferromagnet ${ }^{21}$ with $\mathrm{S}=2$ and $\mathrm{H}=$ $-\mathrm{J} \sum_{i, j} S_{i} S_{j}$. The resulting parameters are $\mathrm{J}=-19 \pm 1 \mathrm{~cm}^{-1}$ and $\mathrm{g}=2.06 \pm 0.3$.
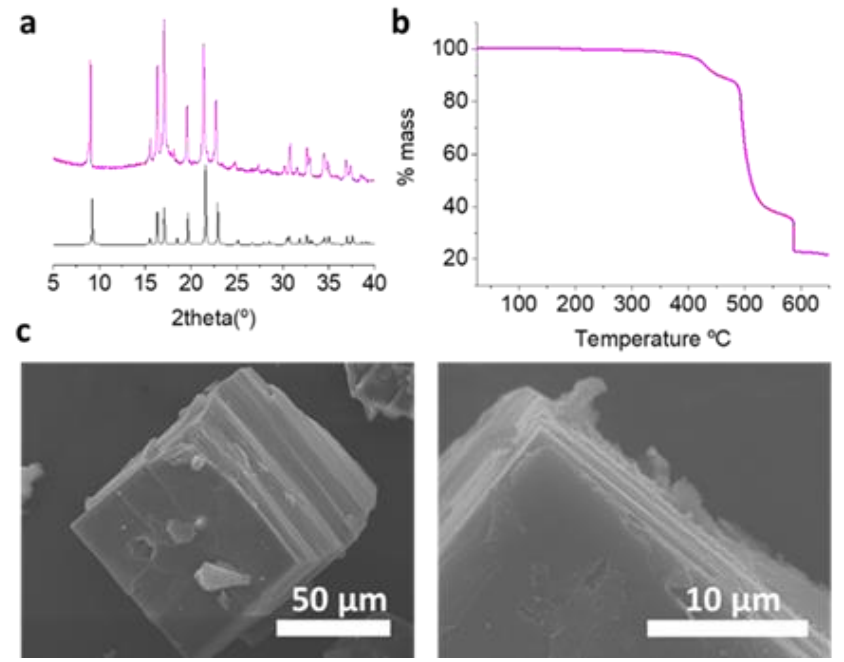

Fig. 2. a) X-ray powder patterns of MUV-1-F. The experimental pattern is shown in magenta and the calculated pattern from 
single-crystal data is shown in black. b) Thermogravimetric analysis of the MUV-1- $F$ at a heating rate of $5{ }^{\circ} \mathrm{C}$ min-1 c) Scanning electron micrographs of bulk-type MUV-1-F.

The Neel temperature and exchange coupling are in the range of those obtained for the other members of the MUV-1-X family $\left(\mathrm{X}=\mathrm{Cl}, \mathrm{H}, \mathrm{Br}, \mathrm{NH}_{2}, \mathrm{CH}_{3} \text { and } \mathrm{F}\right)^{8}$, thus demonstrating that the magnetic properties are dominated by the metal center, their connectivity, and the coordination environment, being independent from the functional group, $X$.

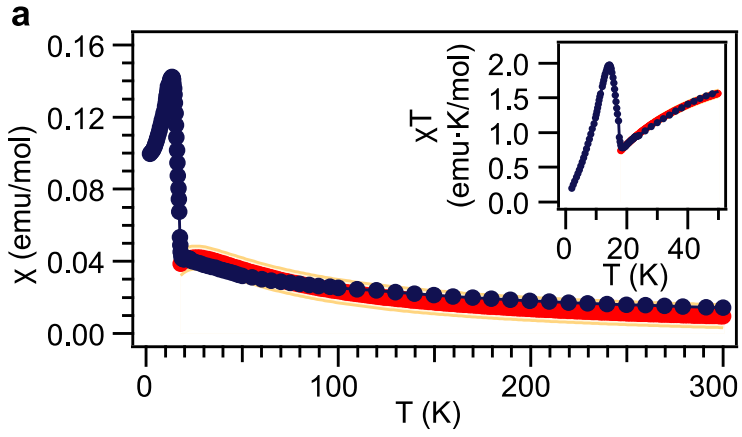

b

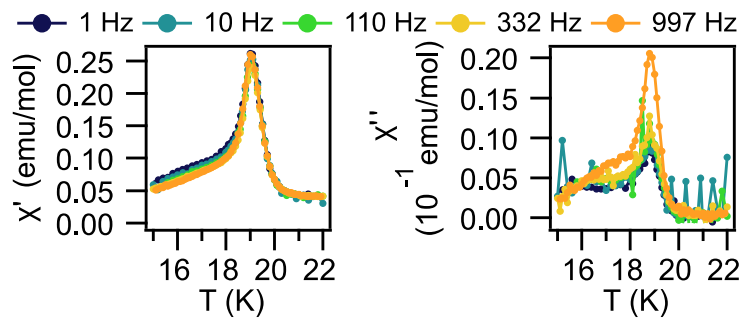

Fig. 3. Magnetic behaviour of MUV-1-F. a) Thermal dependence of $\chi$. Inset: Thermal dependence of the $\chi \top$ product. The data have been fitted (red line) following a Lines expansion for a quadratic-layer Heisenberg antiferromagnet with $S=2 .{ }^{21}$ The red line represents the prediction band with a confidence interval of $95 \%$. b) In-phase (left) and out-of-phase (right) dynamic susceptibility measured at different frequencies.

Finally, we investigated the exfoliation of the MUV-1-F using the micromechanical procedure to preserve the high quality of the crystals. Using the Scotch tape methodology, MUV-1-F was successfully exfoliated. The obtained flakes were deposited onto silicon substrates with $285 \mathrm{~nm}$ of thermally grown $\mathrm{SiO}_{2}$. As a result, a plethora of flakes with remarkable well-defined rectangular shapes (lateral dimensions $>1 \mu \mathrm{m}$ ) and different thicknesses (ranging from few layers, $5 \mathrm{~nm}$, up to hundreds of layers) were obtained (Figure S4). In addition, they were characterized by different microscopic techniques such as atomic force microscopy (Fig. 4a) and transmission electron microscopy (TEM), with the corresponding selected area diffraction pattern, showing crystalline atomically-thin layers with large lateral sizes (several $\mu \mathrm{m}$ ). This is remarkable for 2D molecular compounds, whose lateral sizes are typically in the order of hundreds of nanometers and lack of a complete functionalization coverage. Raman measurements demonstrate the chemical nature compared with the bulk measurements (Fig. 4b) for thin-layers with different thicknesses. In the case of
MUV-1-F, which is chemically more complex than inorganic materials, there is a higher number of vibrations. This factor prevents the high accuracy for detecting changes in the structure. However, we can unambiguously identify the presence of the ligand for all the thin-layers, although with a decrease of the signal intensity with the thickness up to a limit near to $29 \mathrm{~nm}$, revealing that the exfoliated flakes correspond to the material.

a
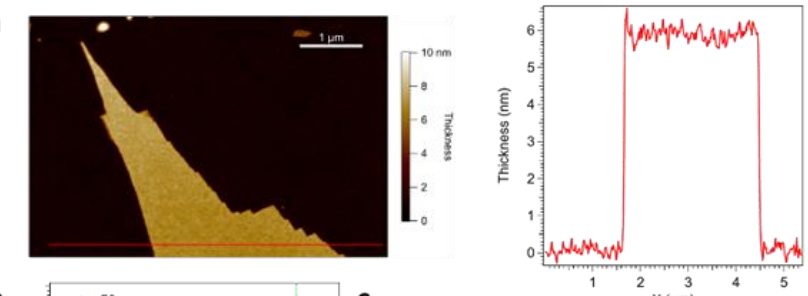

b
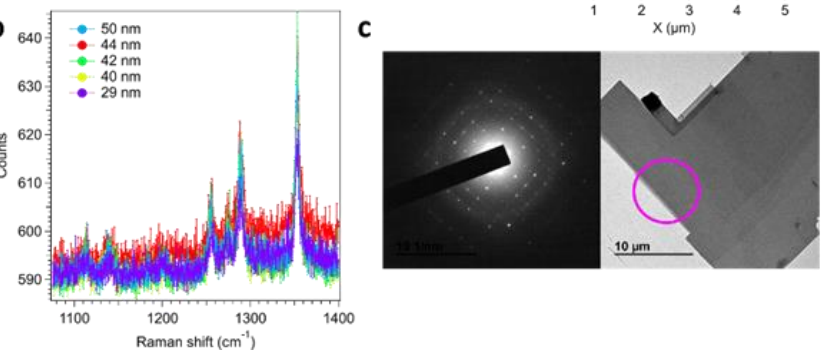

Fig. 4. a) Atomic force microscopy for the MUV-1-F, showing the possibility to achieve few layers thickness with high-quality flakes (lateral size and morphology). b) Raman study of thickness of MUV-1-F flakes. c) TEM image of the diffracted area with its corresponding selected area electron diffraction pattern

\section{Conclusions}

A new 2D magnetic member of the MUV-1 family has been reported, incorporating $\mathrm{F}$ groups at the surface of the layers. The big size, high crystallinity and robustness of the pristine crystals allows to explore the 2D limit. In general, the known 2D inorganic magnetic materials (metal halides, for example) are chemically unstable in open air. The MUV-1 family has shown to be more stable than these inorganic layered materials opening the door to construct mechanical/magnetic devices or to study exotic phenomena in the 2D regime. Still, some oxidation of the $\mathrm{Fe}(\mathrm{II})$ has been detected in the exfoliated layers. Here, the possibility of functionalizing the MUV-1 layers with fluorine has enhanced the stability of these 2D antiferromagnets, while keeping its magnetic properties. This feature may be useful to prepare van der Waals heterostructures based on these molecular magnetic layers, a possibility that so far has only been exploited in inorganic layers.

\section{Experimental section}

\section{Synthesis of MUV-1-F}

Ferrocene $(30 \mathrm{mg}, 0.16 \mathrm{mmol}$ ) and 5-fluorobenzimidazole ( 46.3 $\mathrm{mg}, 0.34 \mathrm{mmol}$ ) were combined and sealed under vacuum in a layering tube ( $4 \mathrm{~mm}$ diameter). The mixture was heated at 250 
${ }^{\circ} \mathrm{C}$ for 3 days to obtain crystals suitable for $\mathrm{X}$-ray single-crystal diffraction. The product was allowed to cool to room temperature, and the layering tube was then opened. The unreacted precursors were extracted with acetonitrile and benzene, and the product was isolated as colorless crystals (yield $80 \%$ ). Phase purity was established by X-ray powder diffraction.

\section{Structural characterization.}

Single crystal X-ray diffraction data for MUV-1-F were collected at a temperature of $100 \mathrm{~K}$ using Rigaku FR-X rotating anode (Mo-k $\alpha, \lambda=071073 \AA$ Á), equipped with a Hybrid Photon detector HyPix-6000HE and an Oxford Cryosystems nitrogen flow gas system. Data was measured and reduced using CrysAlisPro suite of programmes. Absorption correction was performed using empirical methods (SCALE3 ABSPACK) based upon symmetryequivalent reflections combined with measurements at different azimuthal angles. ${ }^{22-24}$ The crystal structures were solved and refined against all $\mathrm{F}^{2}$ values using the SHELXL and Olex 2 suite of programmes. ${ }^{25,26}$ Despite that the coordination polymers are intrinsically chiral, the centrosymmetric space group $C 2 / c$ was found as result of the racemic distribution of the disordered layers of the MUV-1-F coordination polymer, as also observed for MUV-1-H, MUV-1-Cl, MUV-1- $\mathrm{CH}_{3}$ and MUV-1$\mathrm{NH}_{2}{ }^{8}$

$\mathrm{X}$-ray powder diffraction and thermogravimetric analysis A polycrystalline sample of MUV-1-F was lightly ground in an agate mortar and pestle and used to fill a $0.5 \mathrm{~mm}$ borosilicate capillariy that was mounted and aligned on an Empyrean PANalytical powder diffractometer, using $\mathrm{Cu} K \alpha$ radiation $(\lambda=$ $1.54056 \AA$ A). Three repeated measurements were collected at room temperature $\left(2 \theta=5-30^{\circ}\right)$ and merged in a single diffractogram. Thermogravimetric analysis of MUV-1-F was carried out with a Mettler Toledo TGA/SDTA851e/SF/1100 apparatus in the $25-600{ }^{\circ} \mathrm{C}$ temperature range under a $20^{\circ} \mathrm{C} \cdot \mathrm{min}^{-1} \mathrm{scan}$ rate and an air flow of $30 \mathrm{~mL} \cdot \mathrm{min}^{-1}$.

\section{Microscopic characterization}

Scanning Electronic Micrographs were recorded in a Hitachi S4800. Optical images were obtained with a NIKON Eclipse LV100 Optical microscope and AFM images were performed with a Nanoscope IVa Multimode Scanning Probe Microscope (Bruker, Karlsruhe, Germany) in tapping mode. Several mechanical exfoliated flakes were transferred onto a grid with a membrane of amorphous SiN (50 nm thick) using a dry and deterministic method (that involves the use of a micromanipulator and PDMS/PPC polymers, as reported in ref. ${ }^{27}$ ). This has been possible thanks to the robustness of MUV1-F, which has allowed a mechanical exfoliation and transfer into silicon nitride membranes for TEM study. TEM images and diffraction patterns were acquired with a JEOL JEM-2100F with a field emission gun operating at $200 \mathrm{kV}$.

\section{Raman spectroscopy}

Raman spectra were acquired with a micro-Raman (model XploRA ONE from Horiba, Kyoto, Japan) with a grating of 2400 $\mathrm{gr} / \mathrm{mm}$, slit of $50 \mu \mathrm{m}$, and hole of $500 \mu \mathrm{m}$. The employed wavelength was $532 \mathrm{~nm}$. The power density of the laser used for spectra measured at $532 \mathrm{~nm}$ was $5.25 \mathrm{~mW} / \mu \mathrm{m}^{2}$ (bulk crystals) and $170 \mu \mathrm{W} / \mu \mathrm{m}^{2}$ (thin-layers).

\section{Magnetic characterization}

Variable-temperature $(2-300 \mathrm{~K})$ direct current $(\mathrm{dc})$ magnetic susceptibility measurements were carried out in applied fields of $1.0 \mathrm{kOe}$ and variable field magnetization measurements up to $\pm 5 \mathrm{~T}$ at $2.0 \mathrm{~K}$. The susceptibility data were corrected from the diamagnetic contributions as deduced by using Pascal's constant tables. Variable-temperature $(16-23 \mathrm{~K})$ alternating current (ac) magnetic susceptibility measurements in a $\pm 4.0 \mathrm{G}$ oscillating field at frequencies in the range of $1-997 \mathrm{~Hz}$ were carried out in a zero dc field.

\section{Conflicts of interest}

There are no conflicts to declare.

\section{Acknowledgements}

This work has been supported by the EU (ERC Advanced Grant MOL-2D 788222 and ERC Consolidator Grant S-CAGE 724681), grants PID2020-117177GB-I00, PID2020-117152RB-I00 and CEX2019-000919-M, funded by MCIN/AEI/10.13039/501100011033, and the Generalitat Valenciana (PROMETEO program, IDIFEDER/2018/061 and iDiFEDER/2020/063). J.L.-C. acknowledges the Universitat de València for an "Atracció de Talent" fellowship.

\section{References}

1 G. Bottari, M. Ángeles Herranz, L. Wibmer, M. Volland, L. Rodríguez-Pérez, D. M. Guldi, A. Hirsch, N. Martín, F. D'Souza and T. Torres, Chem. Soc. Rev., 2017, 46, 44644500.

X. S. Chu, A. Yousaf, D. O. Li, A. A. Tang, A. Debnath, D. Ma, A. A. Green, E. J. G. Santos and Q. H. Wang, Chem. Mater., 2018, 30, 2112-2128. J. J. Navarro, F. Calleja, R. Miranda, E. M. Pérez and A. L. Vázquez De Parga, Chem. Commun., 2017, 53, 1041810421. L. Assies, C. Fu, P. Kovaříček, Z. Bastl, K. A. Drogowska, J. Lang, V. L. P. Guerra, P. Samorì, E. Orgiu, D. F. Perepichka and M. Kalbáč, J. Mater. Chem. C, 2019, 7, 12240-12247. M. Vera-Hidalgo, E. Giovanelli, C. Navío and E. M. Pérez, J. Am. Chem. Soc., 2019, 141, 3767-3771. I. Gómez-Muñoz, S. Laghouati, R. Torres-Cavanillas, M. Morant-Giner, N. V Vassilyeva, A. Forment-Aliaga and M. Giménez-Marqués, ACS Appl. Mater. Interfaces, 2021, 13, 36475-36481.

7 L. Daukiya, J. Seibel and S. De Feyter, Adv. Phys. X, 2019, 4, 1625723.

J. López-Cabrelles, S. Mañas-Valero, I. J. Vitórica-Yrezábal, P. J. Bereciartua, J. A. Rodríguez-Velamazán, J. C. Waerenborgh, B. J. C. Vieira, D. Davidovikj, P. G. Steeneken, H. S. J. van der Zant, G. Mínguez Espallargas and E. Coronado, Nat. Chem., 2018, 10, 1001-1007. D. D. Chronopoulos, A. Bakandritsos, M. Pykal, R. Zbořil and M. Otyepka, Appl. Mater. Today, 2017, 9, 60-70. 

2019, 6.

O. M. Yaghi, H. Furukawa, W. Morris, C. J. Doonan and R. Banerjee, J. Am. Chem. Soc., 2008, 130, 12626-12627. O. Karagiaridi, M. B. Lalonde, W. Bury, A. a. Sarjeant, O. K. Farha and J. T. Hupp, J. Am. Chem. Soc., 2012, 134, 1879018796.

14 M. Giménez-Marqués, E. Bellido, T. Berthelot, T. SimónYarza, T. Hidalgo, R. Simón-Vázquez, Á. GonzálezFernández, J. Avila, M. C. Asensio, R. Gref, P. Couvreur, C. Serre and P. Horcajada, Small, 2018, 14, 1870182. M. Eddaoudi, Science, 2002, 295, 469-472.

A. M. Fracaroli, H. Furukawa, M. Suzuki, M. Dodd, S. Okajima, F. Gándara, J. A. Reimer and O. M. Yaghi, J. Am. Chem. Soc., 2014, 136, 8863-8866.

J. López-Cabrelles, S. Mañas-Valero, I. J. Vitórica-Yrezábal, M. Šiškins, M. Lee, P. G. Steeneken, H. S. J. van der Zant, G. Mínguez Espallargas and E. Coronado, J. Am. Chem. Soc., 2021, jacs.1c07802. J. López-Cabrelles, J. Romero, G. Abellán, M. GiménezMarqués, M. Palomino, S. Valencia, F. Rey and G. Mínguez Espallargas, J. Am. Chem. Soc., 2019, 141, 7173-7180. F. Hof, R. A. Schäfer, C. Weiss, F. Hauke and A. Hirsch, Chem. - A Eur. J., 2014, 20, 16644-16651. K. Jayaramulu, F. Geyer, A. Schneemann, Š. Kment, M. Otyepka, R. Zboril, D. Vollmer and R. A. Fischer, Adv. Mater., 2019, 31, 1-31. M. E. Lines, J. Phys. Chem. Solids, 1970, 31, 101-116. L. Krause, R. Herbst-Irmer, G. M. Sheldrick and D. Stalke, J. Appl. Crystallogr., 2015, 48, 3-10. R. H. Blessing, Acta Crystallogr. Sect. A Found. Crystallogr., 1995, 51, 33-38. G. M. Sheldrick, 1994, University of Göttingen, Göttingen. G. M. Sheldrick, Acta Crystallogr. Sect. C Struct. Chem., 2015, 71, 3-8. O. V. Dolomanov, L. J. Bourhis, R. J. Gildea, J. A. K. Howard and H. Puschmann, J. Appl. Crystallogr., 2009, 42, 339-341. L. Nguyen, H. P. Komsa, E. Khestanova, R. J. Kashtiban, J. J. P. Peters, S. Lawlor, A. M. Sanchez, J. Sloan, R. V.

Gorbachev, I. V. Grigorieva, A. V. Krasheninnikov and S. J. Haigh, ACS Nano, 2017, 11, 2894-2904. 